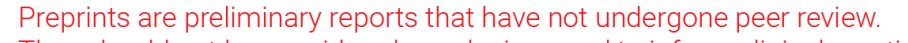 \\ They should not be considered conclusive, used to inform clinical practice, or referenced by the media as validated information. \\ The Key Role of NF-kB Signaling Pathway in Mediating Cholesterol Efflux and Pyroptosis in ox- LDL-induced Macrophages-derived Foam Cells
}

\section{Jiashan Li}

First Affiliated Hospital of Harbin Medical University

Jiaru Liu

First Affiliated Hospital of Harbin Medical University

Ying Yu

First Affiliated Hospital of Harbin Medical University

Yuee Liu

First Affiliated Hospital of Harbin Medical University

Xiuru Guan ( $\nabla$ gxr0451@sina.com )

First Affiliated Hospital of Harbin Medical University https://orcid.org/0000-0001-6024-059X

\section{Research Article}

Keywords: NF-KB signal, ABCA1, atherosclerosis, cell pyroptosis

Posted Date: February 2nd, 2022

DOI: https://doi.org/10.21203/rs.3.rs-1243783/v1

License: (9) This work is licensed under a Creative Commons Attribution 4.0 International License. Read Full License 


\section{Abstract}

Objective NF-KB plays a well-known function in the regulation of immune responses and inflammation, but growing evidences support a major role in atherosclerosis. Currently, the regulatory mechanism of NF$\mathrm{KB}$ pathway involved in atherosclerosis is not clear.

Methods To investigate the role of ox-LDL in NF-KB regulation, the protein expression of phosphorylated NF-KB, a marker of NF-KB pathway activation was measured. The pyroptosis of macrophage was evaluated by western blot and fluorescence microscope. Cholesterol efflux capacity was determined by fluorescence assay and oil red $\mathrm{O}$ staining. The inhibitor of activation of NF-KB signal was used to assess the effect of NF-KB signal on macrophage pyroptosis as well as cholesterol efflux in macrophage. Small interfering RNA of ABCA1 was used to assess the effect of ABCA1 on macrophage pyroptosis.

Results In this study, we reported THP-1 derived macrophage can be stimulated to increase pyroptosis by ox-LDL in a concentration-dependent. Macrophage pyroptosis was correlated with enhanced activation of NF-KB signal. We found that using inhibitor of NF-KB phosphorylation to attenuate activation of NF-KB signal could decrease macrophage pyroptosis and ox-LDL-induced cholesterol efflux

disorder. Furthermore, we found that knocking out of ABCA1 led to increased cell inflammation death. But pyroptosis was blocked, may led to cholesterol efflux dysfunction.

Conclusion This study demonstrates that the mechanism of NF-KB involved in the development of atherosclerosis is depending on mediating cell pyroptosis and cholesterol efflux for the first time and provides significant light on macrophage NF-KB signal in atherosclerosis. In addition, ABCA1 may be involved in the development of atherosclerosis as a bridge between cholesterol efflux and pyroptosis in macrophages.

\section{Key Points}

1. Ox-LDL induced macrophage pyroptosis and the activation of NF-KB signaling in a concentration dependent manner.

2. NF-KB signaling is required for ox-LDL-induced macrophages pyroptosis.

3. NF-KB signal acts as the upstream of $A B C A 1$, and is activated to inhibit the expression of $A B C A 1$ protein.

4. Inhibition of ABCA1 protein expression in THP-1-derived macrophages can increase cell pyroptosis.

\section{Introduction}

Atherosclerosis is a chronic inflammatory disease of artery blood vessels, and the cardiovascular and cerebrovascular diseases caused by it are the leading cause of human death worldwide. However, the pathogenesis of AS is still unclear now. Recent studies have focused on pyroptosis, a unique form and mechanism of programmed cell death compared with apoptosis and necrosis, and characterized by the release of large amounts of inflammatory mediators, which has been shown to be associated with 
atherosclerotic diseases $[18,23]$.Mechanistically, the process of pyroptosis is well preserved across cell types, and characterized by activation of NLRP3 inflammasomes. Activated NLRP3 transduction of the recognition signal into the inflammasome adapter ASC (apoptosis-related SpecK like protein) to activate caspase-1, followed by pro-IL-1 $\beta / 18$ processing and Gasdermin D (GSDMD) cleavage which generates the GSDMD-N terminal[20]. Under electron microscopy, the GSDMD-N terminal causes the formation of cell membrane pores [10], and robust release of proinflammatory cytokines (such as interleukin IL-1 $\beta$ and IL-18). The Gasdermin family represented by GSDMD has the activity of promoting the formation of cell membrane pores. Therefore, pyroptosis is called Gasdermin-regulated programmed cell death $[6,10]$. A large number of studies have shown that cell pyroptosis closely links to the development of AS[4]. Xu S et al. found that nicotine induced pyroptosis of atherosclerotic macrophages through the HDAC6/NFKB/NLRP3 signaling pathway[19]. Zeng Zhaolin et al. have shown that fibroblast growth factor 21(FGF21) alleviates atherosclerosis by inhibiting NLRP3 inflammasome mediated vascular endothelial cell apoptosis [22]. Additionally, recently an important study has reported that antiinflammatory therapy with canakinumab, a therapeutic monoclonal antibody targeting the IL-1 $\beta$ innate immunity pathway led to a significantly lower rate of recurrent cardiovascular events without lowering lipid-level [15].

There are studies showing that GSDMD can be activated by NF-KB signaling[11]. In addition, NF-kB signaling acts as an upstream activation of NLRP3 inflammasome [1], but whether NF-kB signal is involved in ox-LDL-induced pyroptosis still needs to be explored. Additionally, Nuclear Factor Kappa-beta (NF-KB) is recognized as one of the key inflammatory pathways in the chronic diseases such as atherosclerosis. Pang JL et al. have reported that HOX transcript antisense intergenic RNA (HOTAIR) can significantly reduce ox-LDL-induced inflammatory response by inhibiting NF-KB signaling in Raw264.7 cells [14].However, the exact mechanism remains unclear.

Atherosclerosis is closely related to the accumulation of cholesterol in the intima of the arteries. Excess cholesterol is cleared by the reverse cholesterol transport (RCT) pathway, which is the chief antiatherosclerosis mechanism [21]. However, recent studies have shown that blocked cholesterol efflux may promote the activation of NLRP3 inflammasomes, leading to atherogenesis. Cholesterol transporters ATP binding boxes $A 1$ and $\mathrm{G} 1$ (ABCA1/G1) mediate cholesterol transport to HDL, and ABCA1/G1 deficiency in myeloid cells leads to cholesterol accumulation. Studies have shown that deletion of NLRP3 or caspase1/11 reduces the size of atherosclerotic lesions in LDLR -/- mice with a myeloid ABCA1 / G1 deficiency [17] .This study demonstrates that NLRP3 inflammasomes play a key role in cholesterol accumulation promoting the development of $A S$, but the regulation mechanism of ABCA1-regulated cholesterol reverse transport in THP-1-derived macrophages on cell pyroptosis still needs further exploration.

In this study, we explored the mechanism of ox-LDL induced THP-1-derived macrophages pyroptosis and the effect of ABCA1-regulated cholesterol reverse transport disorder on pyroptosis, so as to provide a new strategy for the treatment of atherosclerotic disease.

\section{Materials And Method}




\subsection{Materials}

THP-1 mononuclear macrophage cell lines were selected from Beijing Beina Institute of Biotechnology(BNCC). GSDMD, P-IKB, P-NFKB, $\beta$-actin primary antibody, cholesterol efflux fluorescence detection kit and sheep anti-mouse IgG secondary antibody were all from Abcam. Skim milk powder from BD company;NLRP3 primary antibody from Cell Signaling Technology Inc.;Human IL-1 $\beta$ enzyme-linked immunosorbent assay kit from Elabscience;RPMI 1640 and Fetal Bovine Serum from Hyclone;PMA comes from Sigma;ECL luminescent liquid from Beijing Noblad Technology Co., Ltd.Oil red 0 dye from Beijing Solaibao Biological Co., Ltd.The sheep anti-rabbit IgG secondary antibody was obtained from Beijing Zhongshan Jinqiao Biotechnology Co., Ltd.Ox-LDL from Guangzhou Yiyuan Biotechnology Co., Ltd.Color predyeing protein marker from the United States Thermo Company;HoechST33342 /PI double dyeing kit and lactate dehydrogenase (LDH) assay kit were from Nanjing Jiancheng Institute of Biological Engineering;Cell Counting Kit-8 (CCK-8) was obtained from Tongren Chemical Research Institute, Japan;Bay11-7082 and VX-765 from Shanghai Taosu Biochemical Technology Co., Ltd.SIABCA1 transfection reagent package was obtained from Suzhou Genepharma gene.BCA protein quantitative kit is from China Biyuntian Biotechnology Co., Ltd.

\subsection{Method}

\subsubsection{Cell treatment}

THP-1 monocytes were purchased from the BeNa Culture Collection and cultured in RPMI1640 medium containing $10 \%$ fetal bovine serum (FBS) (both from Hyclone USA) at $37 \mathrm{C}$ and $5 \% \mathrm{CO} 2$. The cells were transferred to 6 -well at a cell density of about $5 \times 10^{5}$. Each well was incubated with PMA (Sigma, USA) at a final concentration of $100 \mathrm{ng} / \mathrm{ml}$ for $48 \mathrm{~h}$ to induce the differentiation of THP-1 cells into macrophages before being treated with the following: ox-LDL $(0,25,50,75$, or $100 \mathrm{mg} / \mathrm{mL})$ for $24 \mathrm{~h}$; or BAY $11-7082$ $(10 \mu \mathrm{M}), \mathrm{VX}-765(50 \mu \mathrm{M})$ for $2 \mathrm{~h}$.

\subsubsection{Western blot analysis}

The total protein was extracted using lysis buffer containing the protease inhibitor PMSF (Beyotime, China), and the protein concentration was measured using the BCA kit (Solarbio, China). The denatured proteins were separated using SDS-polyacrylamide gel electrophoresis, transferred to PVDF membranes, and then blocked with $5 \%$ bovine serum albumin (BSA) for $1 \mathrm{~h}$. Target bands were incubated with the primary antibody overnight at $4 \mathrm{C}$ and then incubated with AP or HRP-conjugated secondary antibody for 1h. The immune complexes were detected in an exposure apparatus using an enhanced chemiluminescence agent, and the target strip protein content was quantified using Tanon Gis software. The dilution ratios for the various antibodies were as follows: ABCA1 (1:500), NLRP3(1:500),GSDMD(1:500), p-NFKB(1:1000), p-IKB(1:1000),b-actin (1:6000), goat anti-mouse secondary antibody (1:10000), and goat anti-rabbit secondary antibody (1:50000).

\subsubsection{Cholesterol efflux detection}


Macrophages were treated with the fluorescence labeling reagent according to the manufacturer's kit instructions in 96 -well plates for 16 hours.After discarding labeling reagent,the cells were gently washed with serum-free RPMI medium and then treated with cholesterol receptor for 4-6 hours. Then the supernatant was removed, $100 \mu \mathrm{l}$ cell lysis buffer was added to each well and the plates were incubated on a shaker for 30 minutes to dissolve the cells. The fluorescence intensity of the supernatant and lysate were measured at an Ex/Em of $482 / 515 \mathrm{~nm}$. The fluorescence intensity of the supernatant was divided by the total fluorescence intensity of the supernatant and the lysate, and the value was multiplied by 100 to obtain a \% cholesterol efflux rate.

\subsubsection{Oil red 0 staining}

The supernatant in the six-well plate was aspirated, then the cells were washed 3 times with PBS and treated with $4 \%$ paraformaldehyde for $6 \mathrm{~min}$. Then remove the paraformaldehyde solution and wash with PBS for 3 times. Add the prepared dilute solution of oil red 0 to the cells at $37^{\circ} \mathrm{C}$ for $15 \mathrm{minThen}$ remove oil red 0 and wash cells with PBS for 3 times. And then you have $60 \%$ isopropanol for 30 s, and washed another three times with PBS for microscopic observation and photography. The PBS in the plate was discarded and the dye was extracted with $100 \%$ isopropyl alcohol for $10 \mathrm{~min}$. The OD value of the mixture was measured at $520 \mathrm{~nm}$ for quantitative analysis.

\subsubsection{Enzyme Linked Immunosorbent Assay}

An appropriate amount of cell culture medium was taken, centrifuged at $1000 \mathrm{~g}$ for 20 minutes, and the supernatant was reserved at $-20^{\circ} \mathrm{C}$ for later use. First set standard hole and blank hole to draw standard curve. Incubate the remaining Wells with $100 \mu \mathrm{l}$ of sample at $37^{\circ} \mathrm{C}$ for $1 \mathrm{~h}$, then remove the sample. Each well was quickly added with working solution $A$ and incubated at $37^{\circ} \mathrm{C}$ for $1 \mathrm{~h}$. Repeat washing the board 3 times and removing. Then, each well was quickly added with working solution $\mathrm{B}$ and incubated at $37^{\circ} \mathrm{C}$ for $30 \mathrm{~min}$. Again wash the plate 5 times and try to drain liquid in the hole. TMB $90 \mu \mathrm{l}$ was added into the corresponding well and incubated at $37^{\circ} \mathrm{C}$ for $15 \mathrm{~min}$. Add $50 \mu \mathrm{l}$ termination solution to the corresponding hole, and record the O.D of each hole at $450 \mathrm{~nm}$ wavelength.Value.

\subsubsection{Determination of LDH (lactate dehydrogenase) content}

Three groups of duplicate holes were set up in the 96-well plate, respectively, for the control hole and the sample hole.5$\mu \mathrm{l}$ of double distilled water was added to each control well, and $20 \mu \mathrm{l}$ of sample and $25 \mu \mathrm{l}$ of matrix buffer were added to each control well and test well. Finally, $5 \mu l$ coenzyme I was added to the experimental well, and the plate was mixed and incubated at $37^{\circ} \mathrm{C}$ for $15 \mathrm{~min}$.After that, $25 \mu \mathrm{l}$ of 4 dinitrophenylhydrazine were added to each well at $37^{\circ} \mathrm{C}$ for $15 \mathrm{~min}$.After discarding liquid, $250 \mu \mathrm{l}$ of $0.4 \mathrm{~mol} / \mathrm{NaOH}$ solution was added to each well at room temperature for $5 \mathrm{~min}$. At the wavelength of $450 \mathrm{~nm}$, the absorbance was measured with a microplate analyzer.

\subsubsection{Hoechst33342/PI double staining test}


The adherent cultured cells were digested and collected by trypsin, then the trypsin was removed by

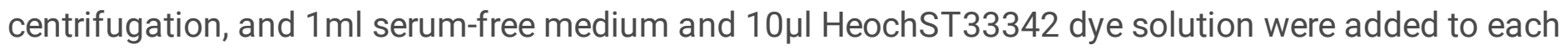
well, mixed, and incubated at $37^{\circ} \mathrm{C}$ for $5-15 \mathrm{~min}$. Then centrifuge at $4^{\circ} \mathrm{C}$ at $800 \mathrm{RPM}$ for 5 minutes to discard the waste liquid; Add $1 \mathrm{ml}$ Buffer $\mathrm{A}$ working solution, suspend cells and add $5 \mu \mathrm{PI}$ dye and avoid light at room temperature for 5-15 minutes. Finally, fluorescence microscope and flow cytometry analysis: PI produced red fluorescence.

\subsubsection{CCK8 cell activity assay}

After cells were treated, the original medium was discarded, $100 \mu \mathrm{l}$ Cell Counting Kit-8 reagent of 10 $\mathrm{mg} / \mathrm{mL}$ was added to each well, and the cells were incubated at $37^{\circ} \mathrm{C}$ for 4 hours. Measure the optical density (OD) value at $450 \mathrm{~nm}$. Each experiment was repeated three times.

\subsubsection{Statistical analysis}

Data were processed using GraphPad Prism 9 software and are expressed as mean \pm standard deviation. The t-test was performed for two-group comparisons, and comparisons of three groups or more were analyzed by one-way analysis of variance. Differences were considered statistically significant at * $p$ $<0.05$.

\section{Results}

\subsection{With the increase of ox-LDL concentrations, macrophage-derived foam cells pyroptosis and NF-KB signal activation both increased}

To test the effect of ox-LDL on the survival rate of macrophages, THP-1-derived macrophages were treated with a range of concentrations of ox-LDL for $24 \mathrm{~h}$. We found cell viability decreased with increasing ox-LDL concentration (Fig. 1a), which indicated a gradual increase in cell death. The pyroptosis of THP-1-derived cells was gradually induced by the increase of ox-LDL concentration as shown by the increased expression of pyroptosis marker protein NLRP3 inflammasome and GSDMD(Fig. $1 \mathrm{bcd}$ ), the increased release of mature IL-1 $\beta$ and LDH in the culture supernatant(Fig-1gh), and the increase of the proportion of Hoechst33342/PI-stained double positive cells(Fig. 1i). Additionally, we found that the expressions of p-NF-кB and p-ІкB proteins were increased, indicating the increased activation of NF-KB signal in a concentration-dependent manner of ox-LDL in macrophages (Figure 1ef).

\subsection{NF-kB phosphorylation inhibitors blocked ox-LDL- induced cell pyroptosis and enhanced ABCA1 protein expression}

BAY11-7082, a specific inhibitor of NF-kB phosphorylation, was cultured with THP-1-derived macrophages at $10 \mu \mathrm{M}$ for $2 \mathrm{~h}$, and then $50 \mu \mathrm{g} / \mathrm{mL}$ ox-LDL was added (according to Figure $1,50 \mu \mathrm{g} / \mathrm{ml}$ ox- 
LDL significantly induced pyroptosis compared with the untreated control group and decreased cell toxic death compared with $75 / 100 \mu \mathrm{g} / \mathrm{mL}$ ox-LDL, so in the following experiments, the macrophages were treated with $50 \mu \mathrm{g} / \mathrm{mL}$ ox-LDL for $24 \mathrm{~h}$ ). We found that the inhibitor was effective as shown by the significantly decreased expression of p-NFKB in the experimental group (Fig. 2AB). Meanwhile, the protein expression of NLRP3 and GSDMD as well as the IL-1 $\beta$ contents in the medium (Fig. 2E) were also significantly decreased (Fig. 2ACD). And the results of Hoechst33342/PI double staining and LDH release assay also showed that the formation of cell membrane pores and the release of cellular contents reduced (Fig. 2FJ). In summary, we found that inhibition of NF-kB signal activation could significantly block ox-LDL-induced cell pyroptosis.

Surprisingly, ox-LDL may inhibit ABCA1-regulated reverse cholesterol transport by activating NF-KB signal as shown by increased ABCA1 expression (Fig. 2GH) and cholesterol efflux (Figure 2I) under BAY11-7082 treatment, which significantly reversed the inhibition of ox-LDL treatment on the expression of $A B C A 1$ proteins and ABCA1-regulated cholesterol efflux.

\subsection{Dysfunction of ABCA1-regulated cholesterol efflux increases cell pyroptosis}

First, The three kinds of SiABCA1 were mixed with transfection reagents for treatment of macrophages for 5h, and siABCA1(a) with the best inhibition effect was selected by Western Blot(Fig. 3ab). Therefore, in the following experiment, si-ABCA1 (a) was used to treat macrophages.

Si-control and Si-ABCA1 transfection reagent mixture was added into the medium and cultured THP -1 derived macrophages at $37^{\circ} \mathrm{C}$ for $5 \mathrm{~h}$,then cells were treated with $50 \mu \mathrm{g} / \mathrm{mL}$ ox-LDL for $24 \mathrm{~h}$. Further tests showed increased the degree of cell foaming and blocked cholesterol efflux (Figure 3ced). In addition, we also found that the transfected siABCA1 reagent was effective as shown by the inhibition of ABCA1 protein expression (Fig. 3g). And the expression of NLRP3 inflammasome and GSDMD (Fig. 3fhi) proteins increased. At the same time, increased levels of LDH (Fig. 3k), mature IL-1 $\beta$ (Figure 3j) in the medium and the increased proportion of positive cells after Hoechst33342/PI double staining also indicated that si-ABCA1 treatment can increase cell pyroptosis, which is characterized by cell pore formation (Fig. 3l).These results suggested that ABCA1-regulated blocking of cholesterol efflux led to increased cell pyroptosis in THP-1-derived macrophages.

\subsection{Cell pyroptosis is a double-edged sword, and inhibition of cell pyroptosis may increase the cell foaming}

First, macrophages were treated with $50 \mu \mathrm{g} / \mathrm{mL}$ VX-765(a pyroptosis inhibitor) for $2 \mathrm{~h}$, and then treated with $50 \mu \mathrm{g} / \mathrm{mL}$ ox-LDL for $24 \mathrm{~h}$. According to the results, PI-positive cells (Q4 region) characterized by damaged cell membranes were reduced after treatment with VX-765 (Fig. 4ac), which indicated that VX765 is effective as a pyroptosis inhibitor. Then fluorescence assay was used to detect cholesterol efflux in foam cells, and the results showed that after VX-765 treatment, the cholesterol efflux in foam cells was 
blocked (Fig. 4e).At the same time, the lipid droplets in the foam cells were increased and the degree of cell foaming aggravated(Fig. $4 \mathrm{bd}$ ).

\section{Discussion}

Recent studies have demonstrated that the monoclonal IL (interleukin)-1 $\beta$-neutralizing antibody canakinumab significantly lowered rate of recurrent cardiovascular events [15]. The NLRP3 inflammasome, an innate immune signaling complex, is the key mediator of IL-1 family cytokine production in atherosclerosis, which is activated by abundant endogenous danger signals, such as oxidized low density lipoprotein (ox-LDL) and cholesterol crystals [3, 5, 25]. Zhuang et al. found in vivo that KLF2-FOXP13 transcriptional signal network can inhibit the expression of endothelial inflammasome NLRP3, alleviating endothelial inflammation and delay the development of AS [24]. In our study, we found that ox-LDL induced NLRP3 inflammasome mediated macrophages pyroptosis in a concentrationdependent manner. Additionally, the expression of $\mathrm{p}-\mathrm{NFKB}$ and $\mathrm{p}-\mathrm{I} \mathrm{KB}$ proteins, as markers of NF-KB signal activation, was gradually increased with the increase of ox-LDL concentration. Therefore, the above experimental results indicate that ox-LDL-induced NLRP3-mediated cells pyroptosis may be associated with the activation of NF-KB signaling.

Activated NF-KB plays a pivotal role in the formation and stability of atherosclerotic plaques [12]. And in the presence of external risk factors such as high lipid induction, the key step in NF-KB activation is the phosphorylation of $\mathrm{I}_{\mathrm{KBa}}$ protein. Then $\mathrm{p}-\mathrm{I}_{\mathrm{KBa}}$ is ubiquitinated and releases NF-kB dimer, which is further activated by chemical modification such as phosphorylation, and then transported to the nucleus[13]. Liu $Z$ et al. demonstrated that melatonin alleviates adipocyte pyroptosis by blocking NF-KB/GSDMD signaling in mouse adipose tissue[11],which suggests that NF-KB signaling may play a essential role in triggering pyroptosis. But it is not clear whether NF-KB activation has an effect on ox-LDL-induced THP-1 derived macrophage pyroptosis, which requires further study. Our experimental results indicated that inhibition of NF-KB signaling activation can effectively diminish ox-LDL-induced cell pyroptosis via inhibiting the activation of NLRP3 inflammasome, suggesting that NF-KB/NLRP3 pathway was required for ox-LDL induced macrophages pyroptosis, consistent with previous researches[19].

A recent study has shown that NF-KB activation may be associated with ABCA1 expression and cholesterol efflux in macrophages[9]. In our study, we found that NF-KB signaling activation inhibitors significantly blocked ox-LDL inhibition of $A B C A 1$ expression and increased cholesterol efflux in macrophage-derived foam cells, which suggests that under ox-LDL treatment, NF-KB signal acts as the upstream of $A B C A 1$ and is activated by ox-LDL to inhibit the expression of $A B C A 1$, impeding the reverse cholesterol transport, and aggravating the degree of cell foaming.

In 2018, a study showed that cholesterol accumulation in bone marrow cells activates NLRP3 inflammasomes, further contributing to the development of AS plaques [17].Similarly, in myeloid ABCA1/ G1-deficient LDLR -/- mice, the deletion of NLRP3 or caspase-1/11 reduced the size of atherosclerotic lesions [17]. In our study, we demonstrated for the first time in vitro that the inhibition of cholesterol 
reverse transport in macrophage-derived foam cells increases the expression of NLRP3 inflammasome, thereby regulating cell pyroptosis and triggering inflammatory response.

Although some studies have shown that pyroptosis activates the inflammatory network and releases a large number of inflammatory factors, promoting the development of atherosclerosis, which is adverse to the development of AS and the stability of plaques $[18,23]$. However, several studies have shown that cell pyroptosis plays an important role in controlling microbial infection. Costa Franco MMS et al. found that in the dendritic cells of C57BL/ 6 mice infected with Brucella, the genomic DNA of Brucella can cause pyroptosis of bone marrow-derived dendritic cells, which has been shown to help control the infection and accelerate the clearance of the pathogen [2]. Wang et al. have recently shown that pyroptosis induced inflammation can trigger a powerful anti-tumor immune effect [16], which is conducive to tumor clearance. The above studies indicate that moderate pyroptosis which is an important immune defense response of the body and plays an important role in resisting infection and endogenous danger signals, can quickly remove pathogens and risk factors, enhancing the immune function[7]. It was previously reported that treatment of smooth muscle cells with VX-765 at 50 $\mu \mathrm{M}$ effectively reduced pyroptosis[8]. Therefore, in this study, we treated macrophages with $50 \mu \mathrm{MVX}-765$, and found that ox-LDL-induced cell pyroptosis was significantly reduced. We also found for the first time that the accumulation of lipid droplets in foam cells was higher, and the intracellular cholesterol efflux was decreased, suggesting that cell pyroptosis may be a double-edged sword in the development of AS.

In conclusion, NF-KB pathway aggravation of ox-LDL-induced cell pyroptosis and cholesterol efflux disorder has been demonstrated in THP-1-derived macrophages for the first time, providing a novel therapeutic avenue for the treatment of AS.

\section{Declarations}

\section{Ethical statement}

Not applicable.

\section{Compliance with ethical standards}

\section{Conflict of interest}

The authors have no conflicts of interest to declare that are relevant to the content of this article.

\section{Research involving Human Participants and/or Animals}

Not applicable.

\section{Informed consent}

Not applicable. 


\section{Funding}

The research was supported by the National Natural Science Foundation of China (81672084).

\section{Authors Contributions}

Jiashan Li and Xiuru Guan: conception, design and analysis of data, performed the data analyses, and wrote the manuscript. Jiashan Li,Jiaru Liu and Ying Yu: contributed to the conception of the study. Jiashan Li and Yuee Liu: contributed signifcantly to analysis and manuscript preparation.

\section{Data availability statement}

My manuscript has no associated data.

\section{Code availability}

None

\section{References}

1. Chen X, Liu G, Yuan Y, Wu G, Wang S, Yuan L Nek7 interacts with nlrp3 to modulate the pyroptosis in inflammatory bowel disease via nf-kappab signaling. Cell Death Dis.2019; 10: 906.https:// DOI: 10.1038/s41419-019-2157-1.

2. Costa Franco MMS, Marim FM, Alves-Silva J, Cerqueira D, Rungue M, Tavares IP, Oliveira SC Aim2 senses brucella abortus DNA in dendritic cells to induce il-1 beta secretion, pyroptosis and resistance to bacterial infection in mice. Microbes Infect.2019; 21: 85-93.https:// DOI:

10.1016/j.micinf.2018.09.001.

3. Grebe A, Hoss F, Latz E Nlrp3 inflammasome and the il-1 pathway in atherosclerosis. Circ Res.2018; 122: 1722-1740.https:// DOI: 10.1161/CIRCRESAHA.118.311362.

4. He B, Nie Q, Wang F, Han Y, Yang B, Sun M, Fan X, Ye Z, Liu P, Wen J Role of pyroptosis in atherosclerosis and its therapeutic implications. J Cell Physiol.2021; DOI: 10.1002/jcp.30366.

5. Hoseini Z, Sepahvand F, Rashidi B, Sahebkar A, Masoudifar A, Mirzaei H Nlrp3 inflammasome: Its regulation and involvement in atherosclerosis. J Cell Physiol.2018; 233: 2116-2132.https:// DOI: 10.1002/jcp.25930.

6. Jia C, Chen H, Zhang J, Zhou K, Zhuge Y, Niu C, Qiu J, Rong X, Shi Z, Xiao J, Shi Y, Chu M Role of pyroptosis in cardiovascular diseases. Int Immunopharmacol.2019; 67: 311-318.https:// DOI: 10.1016/j.intimp.2018.12.028.

7. Jorgensen I, Rayamajhi M, Miao EA Programmed cell death as a defence against infection. Nat Rev Immunol.2017; 17: 151-164.https:// DOI: 10.1038/nri.2016.147.

8. Li Y, Niu X, Xu H, Li Q, Meng L, He M, Zhang J, Zhang Z, Zhang Z Vx-765 attenuates atherosclerosis in apoe deficient mice by modulating vsmcs pyroptosis. Exp Cell Res.2020; 389: 111847.https:// DOI: 
10.1016/j.yexcr.2020.111847.

9. Li Y, Shen S, Ding S, Wang L Toll-like receptor 2 downregulates the cholesterol efflux by activating the nuclear factor-kappab pathway in macrophages and may be a potential therapeutic target for the prevention of atherosclerosis. Exp Ther Med.2018; 15: 198-204.https:// DOI: 10.3892/etm.2017.5404.

10. Liu X, Zhang Z, Ruan J, Pan Y, Magupalli VG, Wu H, Lieberman J Inflammasome-activated gasdermin d causes pyroptosis by forming membrane pores. Nature.2016; 535: 153-158.https:// DOI:

$10.1038 /$ nature18629.

11. Liu Z, Gan L, Xu Y, Luo D, Ren Q, Wu S, Sun C Melatonin alleviates inflammasome-induced pyroptosis through inhibiting nf-kappab/gsdmd signal in mice adipose tissue. J Pineal Res.2017; 63 DOI: 10.1111/jpi.12414.

12. Meng Z, Hernandez R, Liu J, Gwag T, Lu W, Hsiai TK, Kaul M, Zhou T, Zhou C Hiv protein tat induces macrophage dysfunction and atherosclerosis development in low-density lipoprotein receptordeficient mice. Cardiovasc Drugs Ther.2021; DOI: 10.1007/s10557-021-07141-x.

13. Mitchell JP ,Carmody RJ Nf-kappab and the transcriptional control of inflammation. Int Rev Cell Mol Biol.2018; 335: 41-84.https:// DOI: 10.1016/bs.ircmb.2017.07.007.

14. Pang JL, Wang JW, Hu PY, Jiang JS, Yu C Hotair alleviates ox-ldl-induced inflammatory response in raw264.7 cells via inhibiting nf-kappab pathway. Eur Rev Med Pharmacol Sci.2018; 22: 69916998.https:// DOI: 10.26355/eurrev_201810_16170.

15. Ridker PM, Everett BM, Thuren T, MacFadyen JG, Chang WH, Ballantyne C, Fonseca F, Nicolau J, Koenig W, Anker SD, Kastelein JJP, Cornel JH, Pais P, Pella D, Genest J, Cifkova R, Lorenzatti A, Forster T, Kobalava Z, Vida-Simiti L, Flather M, Shimokawa H, Ogawa H, Dellborg M, Rossi PRF, Troquay RPT, Libby P, Glynn RJ, Group CT Antiinflammatory therapy with canakinumab for atherosclerotic disease. N Engl J Med.2017; 377: 1119-1131.https:// DOI: 10.1056/NEJMoa1707914.

16. Wang Q, Wang Y, Ding J, Wang C, Zhou X, Gao W, Huang H, Shao F, Liu Z A bioorthogonal system reveals antitumour immune function of pyroptosis. Nature.2020; 579: 421-426.https:// DOI: 10.1038/s41586-020-2079-1.

17. Westerterp M, Fotakis P, Ouimet M, Bochem AE, Zhang H, Molusky MM, Wang W, Abramowicz S, la Bastide-van Gemert S, Wang N, Welch CL, Reilly MP, Stroes ES, Moore KJ, Tall AR Cholesterol efflux pathways suppress inflammasome activation, netosis, and atherogenesis. Circulation.2018; 138: 898-912.https:// DOI: 10.1161/CIRCULATIONAHA.117.032636.

18. Xing SS, Yang J, Li WJ, Li J, Chen L, Yang YT, Lei X, Li J, Wang K, Liu X Salidroside decreases atherosclerosis plaque formation via inhibiting endothelial cell pyroptosis. Inflammation.2020; 43: 433-440.https:// DOI: 10.1007/s10753-019-01106-x.

19. Xu S, Chen H, Ni H, Dai Q Targeting hdac6 attenuates nicotine-induced macrophage pyroptosis via nf-kappab/nlrp3 pathway. Atherosclerosis.2021; 317: 1-9. https:// DOI:

10.1016/j.atherosclerosis.2020.11.021.

20. Xu YJ, Zheng L, Hu YW, Wang Q Pyroptosis and its relationship to atherosclerosis. Clin Chim Acta.2018; 476: 28-37.https:// DOI: 10.1016/j.cca.2017.11.005. 
21. Yu XH, Zhang DW, Zheng XL, Tang CK Cholesterol transport system: An integrated cholesterol transport model involved in atherosclerosis. Prog Lipid Res.2019; 73: 65-91.https:// DOI: 10.1016/j.plipres.2018.12.002.

22. Zeng Z, Zheng Q, Chen J, Tan X, Li Q, Ding L, Zhang R, Lin X Fgf21 mitigates atherosclerosis via inhibition of nlrp3 inflammasome-mediated vascular endothelial cells pyroptosis. Exp Cell Res.2020; 393: 112108.https:// DOI: 10.1016/j.yexcr.2020.112108.

23. Zhang Y, Liu X, Bai X, Lin Y, Li Z, Fu J, Li M, Zhao T, Yang H, Xu R, Li J, Ju J, Cai B, Xu C, Yang B Melatonin prevents endothelial cell pyroptosis via regulation of long noncoding rna meg $3 / \mathrm{mir}-$ 223/nlrp3 axis. J Pineal Res.2018; 64 DOI: 10.1111/jpi.12449.

24. Zhuang T, Liu J, Chen X, Zhang L, Pi J, Sun H, Li L, Bauer R, Wang H, Yu Z, Zhang Q, Tomlinson B, Chan P, Zheng X, Morrisey E, Liu Z, Reilly M, Zhang Y Endothelial foxp1 suppresses atherosclerosis via modulation of nlrp3 inflammasome activation. Circ Res.2019; 125: 590-605.https:// DOI: 10.1161/CIRCRESAHA.118.314402.

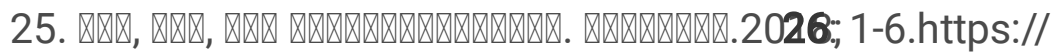

\section{Figures}



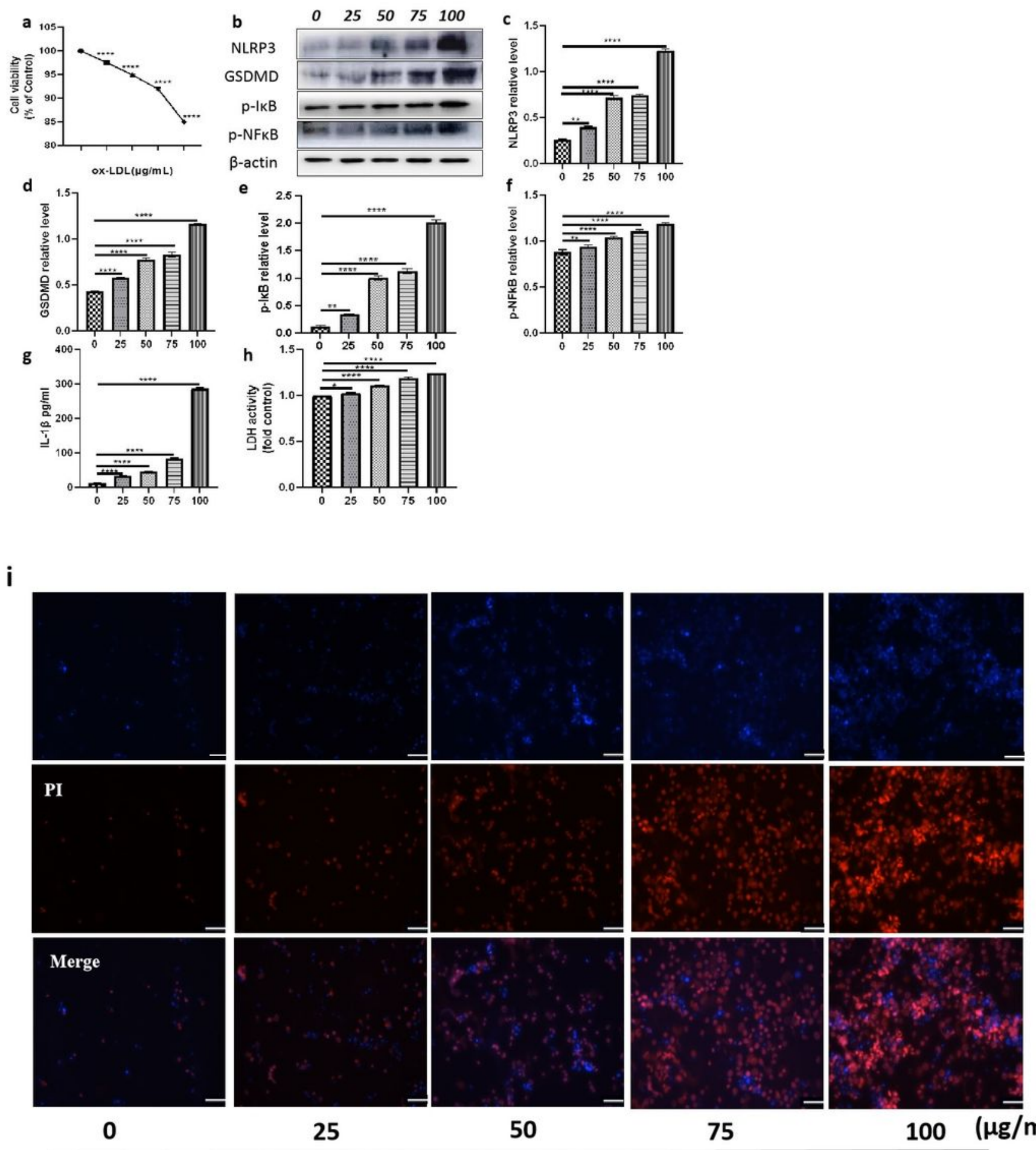

25
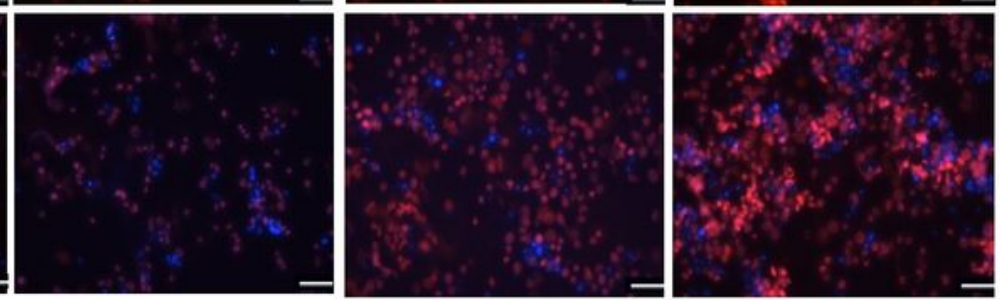

75

$100(\mu \mathrm{g} / \mathrm{ml})$

ox-LDL

Figure 1

With the increase of ox-LDL concentrations, macrophage-derived foam cells pyroptosis and NF-KB signal activation both increased (A) CCK8 assay for cell viability;(B) The expression of NLRP3, GSDMD and p$\mathrm{NFKB}$ and $\mathrm{p}-\mathrm{IKB}$ protein were detected by Western Blot after treatment with ox-LDL concentration gradient $(0,25,50,75,100 \mu \mathrm{g} / \mathrm{mL})$, and $\beta$-actin was used as internal reference.(c-f) Statistical graph of relative gray values of protein bands; (g) ELISA was used to detect the content of IL-1 $\beta$ in the supernatant of culture 
medium;(h) The content of LDH in medium supernatant was detected and analyzed statistically;(I) Fluorescence microscopy analysis after Hoechst33342/PI double staining;the scale is $50 \mu \mathrm{m}$. Among them, * $\mathrm{P}<0.05, * * \mathrm{P}<0.01, * * * \mathrm{P}<0.001, * * * * \mathrm{P}<0.0001, \mathrm{n}=3$.
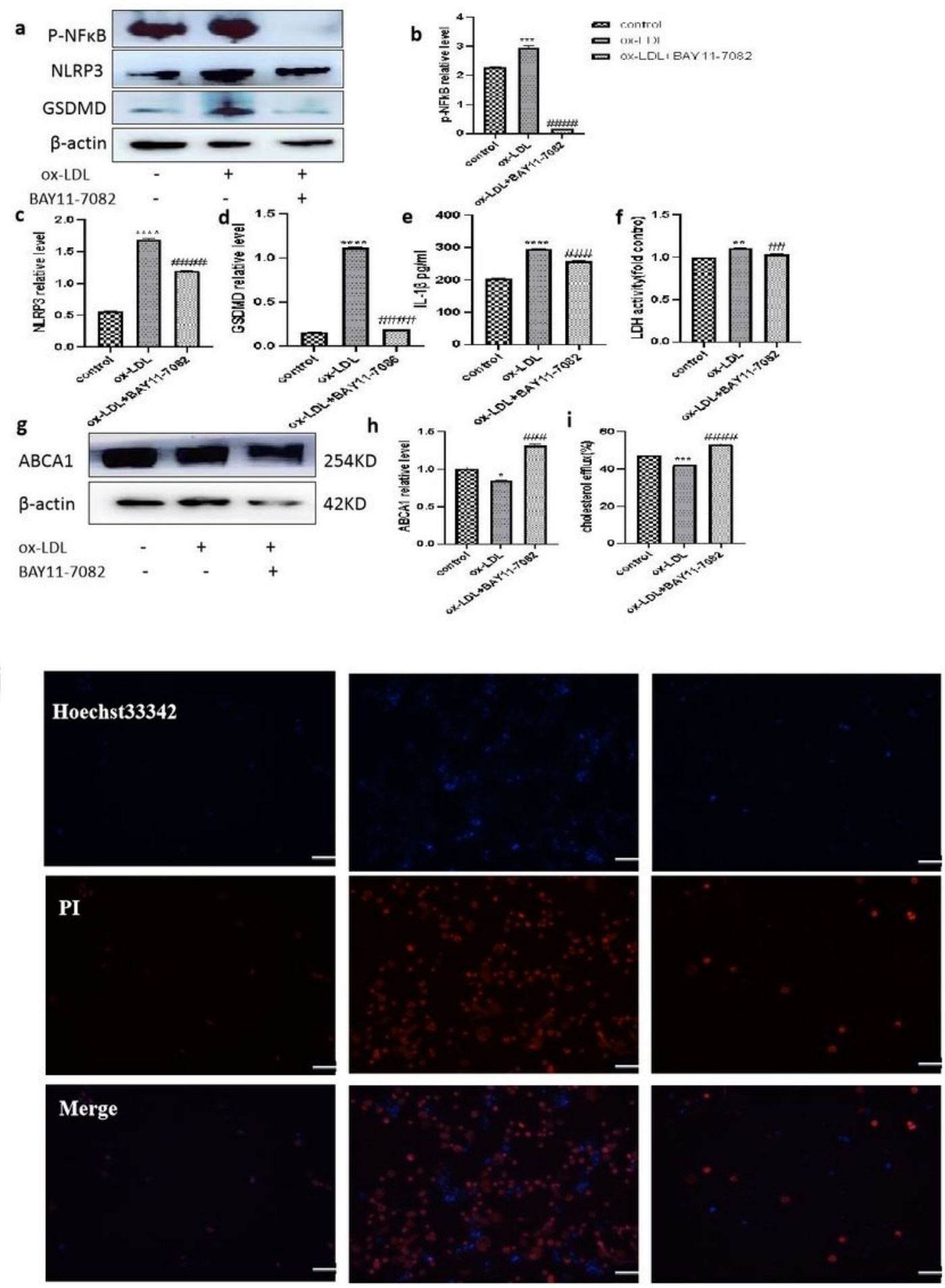

control

$o x-L D L$

$o x-L D L+B A Y 11-7082$

Figure 2 
NF-KB phosphorylation inhibitors block ox-LDL-induced pyrotosis and enhance ABCA1 protein expression.

(A) The protein expressions of p-NF-kB, NLRP3 and GSDMD were detected by Western Blot. (b-d) Statistical graph of the relative gray values of protein bands;(E) ELISA was used to detect the content of IL-1 $\beta$ in the supernatant;(f) Determination of LDH release and histogram of statistical analysis;(J) Fluorescence microscopy analysis after Hoechst33342/PI double staining. The scale is $50 \mu \mathrm{m} ;(\mathrm{G}-\mathrm{H})$ Western Blot analysis of ABCA1 expression and histogram of relative gray value;(I) Measure the effluent capacity of cholesterol in foam cells. * represents Control vs Ox-LDL;\# stands for ox-LDLvsOXLDL+BAY11-7082, *P<0.05** $P<0.01 * * * P<0.001$, the **** $P<0.0001 ; \# P$ in the same way; $N=3$. 

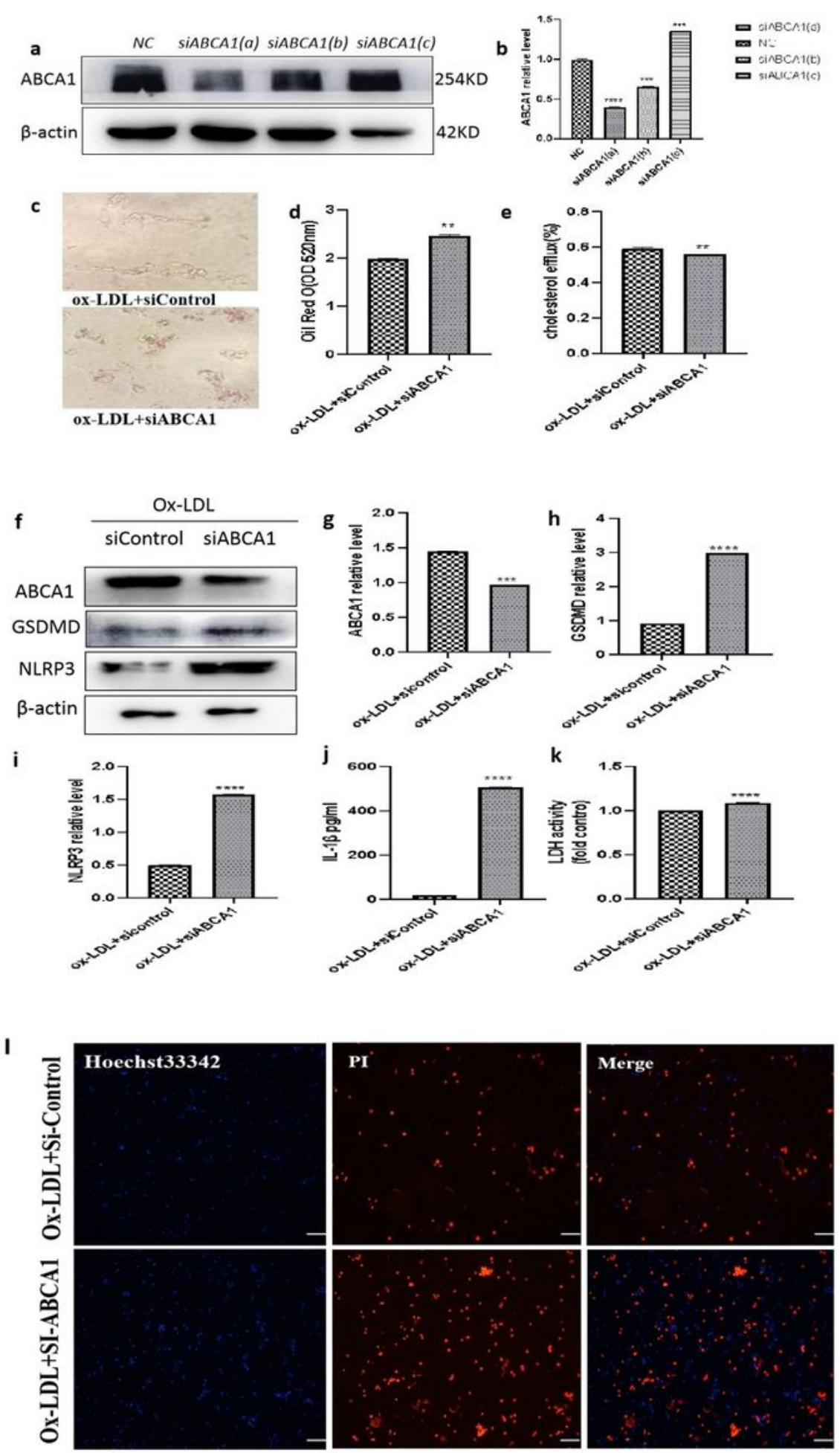

\section{Figure 3}

Dysfunction of ABCA1-regulated cholesterol efflux increases cell pyroptosis. (A-B) The expression of ABCA1 protein was detected by Western Blot and the siABCA1 with the best inhibitory effect was selected.(C-D) Oil red $O$ staining (40×magnification) and intracellular lipid titration detection in each of the treatment groups.(E) Fluorescence reagent was used to detect the effluent capacity of cholesterol in foam cells after SiABCA1 treatment;(F-I) Western Blot analysis of ABCA1, NLRP3 and GSDMD protein 
expression and gray scale of corresponding protein bands;(J) ELISA was used to detect the content of IL$1 \beta$ in the medium; $(K)$ Lactate dehydrogenase release test; $(L)$ Hoechst33342/PI double staining was used to detect cell death;the scale is $50 \mu \mathrm{m}$. Among them, ${ }^{*} \mathrm{P}<0.05, * * \mathrm{P}<0.01, * * * \mathrm{P}<0.001, * * * * \mathrm{P}<0.0001, \mathrm{n}$ $=3$.

a
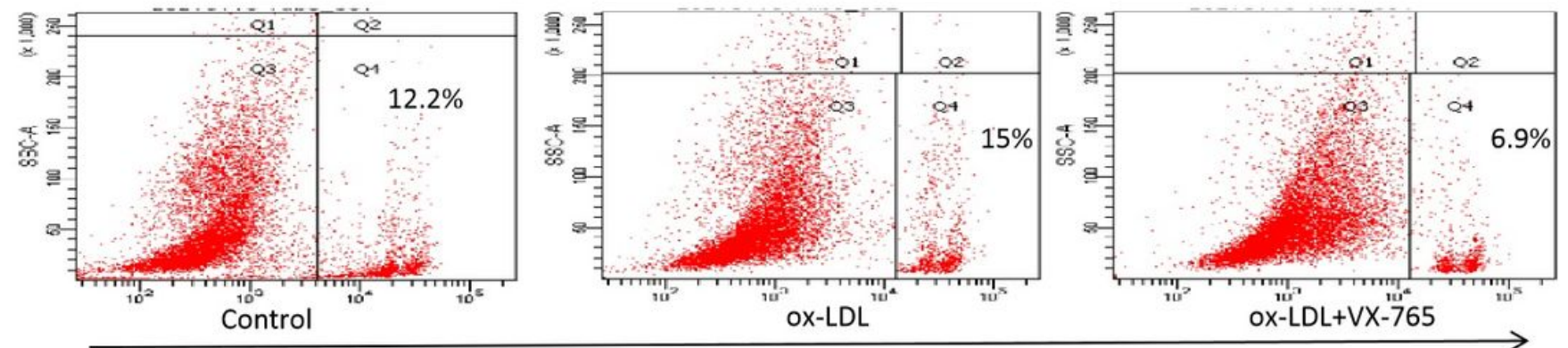

b

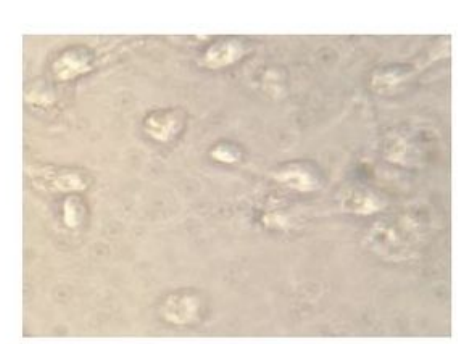

Control

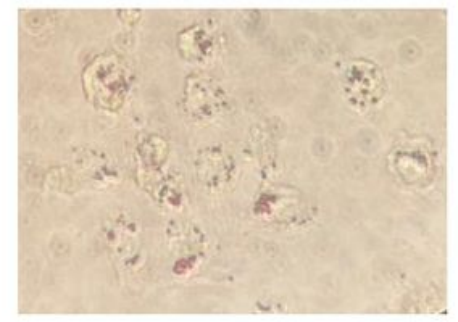

ox-LDL

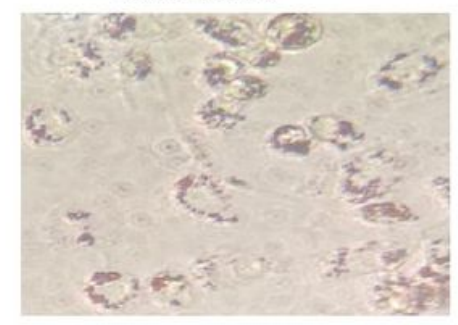

ox-LDL+VX-765

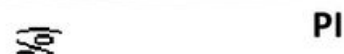

Pl

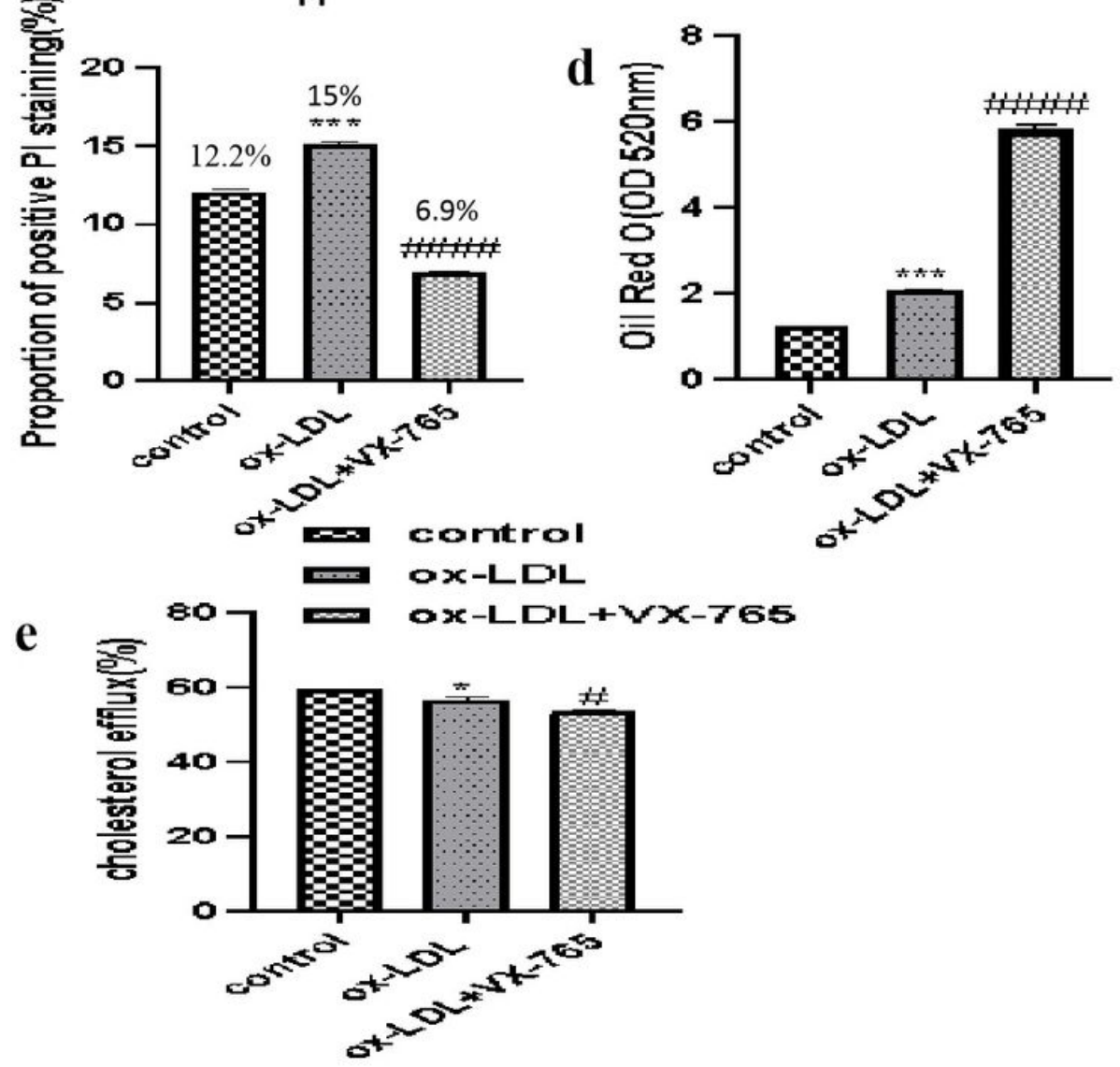

Figure 4

Cell apoptosis is a doubleedged sword. Inhibition of cell apoptosis may increase the form. (A) After treatment, PI staining cell death was detected by flow cytometry;(B.D) Oil red 0 staining(40×magnification) and intracellular lipid titration detection in each of the treatment groups (C) Statistical analysis was made of the positive results of PI staining in each group in Figure 4a. (E) Fluorescence test was used to detect the efflux capacity of cholesterol after treatment with pyroptosis inhibitors. * represents Control vs Ox-LDL; \# stands for Ox-LDL vs OX-LDL +VX-765;*P< 0.05, * * $P<0.01$, * * ${ }^{*} \mathrm{P}<0.001, * * * * \mathrm{P}<0.0001 ; \# \mathrm{P}$ in the same way, $\mathrm{N}=3$. 